\title{
Editorial
}

\section{Can young people reliably rate side effects of low-dose antipsychotic medication using a self-report survey?}

Walter G, Byrne S, Griffiths O, Hunt G, Soh N, Cleary M, Duffy P, Crawford G, Krabman P, Concannon P, Malhi G. Can young people reliably rate side effects of low-dose antipsychotic medication using a self-report survey?

\section{Garry Walter ${ }^{1,2}$, Simon Byrne ${ }^{2}$, Oren Griffiths ${ }^{3}$, Glenn Hunt ${ }^{1,4}$, Nerissa Soh ${ }^{2}$, Michelle Cleary ${ }^{5}$, Paul Duffy ${ }^{6}$, Geoff Crawford ${ }^{7}$, Peter Krabman ${ }^{8,9}$, Patrick Concannon ${ }^{10}$, Gin Malhi ${ }^{1,11}$}

${ }^{1}$ Discipline of Psychiatry, Sydney Medical School, University of Sydney, NSW, Australia; ${ }^{2}$ Child and Adolescent Mental Health Services, Northern Sydney Central Coast Health, NSW, Australia; ${ }^{3}$ School of Psychology, University of New South Wales, NSW, Australia; ${ }^{4}$ Sydney South West Area Mental Health Service, NSW, Australia; ${ }^{5}$ School of Nursing \& Midwifery, University of Western Sydney, Penrith, NSW, Australia; ${ }^{6}$ Private Practice, Dee Why. NSW, Australia; ${ }^{7}$ Private Practice, Sydney, NSW, Australia; ${ }^{8}$ Coral Tree Family Service, Northern Sydney Central Coast Health, North Ryde, NSW Australia; ${ }^{9}$ Private Practice, Eastwood, NSW, Australia; ${ }^{10}$ Private Practice, Chatswood, NSW, Australia; and ${ }^{11}$ CADE Clinic, Northern Clinical School, Royal North Shore Hospital, St Leonards, Sydney, Australia

Professor Garry Walter,

Coral Tree Family Service, Northern Sydney Central Coast Health, P0 Box 142, North Ryde, NSW 1670, Australia.

Tel: +61 29887 5830;

Fax: +61 298872941 ;

E-mail: gwalter@mail.usyd.edu.au
Many neuropsychiatric illnesses commence in adolescence, or sooner, and psychotropic medication plays a key role in the overall treatment. The monitoring and management of side effects from these medications is an important component of psychiatric care in the child and adolescent population. To best achieve this, clinicians are dependent upon reliable self-report of side effects from their young patients.

There has been a noticeable increase in the use of antipsychotic medications to treat psychiatric disorders in young people over the past 10 years $(1,2)$. Low doses of newer and purportedly safer 'atypical antipsychotics' have been used to treat symptoms of a diverse range of child and adolescent psychiatric disorders, including psychoses, bipolar disorder, autism and behavioural problems $(3,4)$. Risperidone is the most commonly prescribed atypical antipsychotic for young people in North America, the United Kingdom and Australia (4-7). Low doses of risperidone have generally been shown to be safe and well tolerated in this population $(8-10)$.

Despite being generally well tolerated, many young people experience adverse effects associated with antipsychotics such as risperidone. Correll (11-13) argued that young people may be more vulnerable to some adverse effects associated 
with antipsychotic medication, such as weight gain, sedation and extrapyramidal symptoms. Side effects from antipsychotic medication may cause discomfort and lead to non-compliance (14). Furthermore, concern has been expressed that these medications have potential to cause serious longer term consequences for children and adolescents' developing minds and bodies $(12,15)$.

The greater sensitivity and potential risk associated with antipsychotic usage in young people highlight the importance of effective side effect monitoring and management in this population. Side effect monitoring is contingent upon a young person's ability to credibly self-report. An important question for clinicians is whether young people can reliably self-report antipsychotic side effects. Similarly, researchers need to establish the credibility of young people's selfreport when investigating the safety and tolerability of these medications in this population.

\section{The LUNSERS: a self-report measure of antipsychotic side effects}

Self-report surveys can be an efficient means for investigators to ascertain a patient's side-effect profile. The Liverpool University Neuroleptic SideEffect Rating Scale [LUNSERS; (16)] consists of 50 five-point Likert scale items asking patients how much a particular side effect has occurred in the last 2 weeks ( 0 'not at all' to 4 'very much'). Male patients can score between 0 and 156 on the LUNSERS and female patients can score between 0 and 164. The LUNSERS can be broken down into seven side-effect subscales: psychic (pertaining to mind and emotion), extrapyramidal, hormonal, anticholinergic, autonomic, allergic reactions and miscellaneous. While a cut-off is somewhat arbitrary, Day et al. (16) suggested that scores of 20 and above indicate potentially significant side effect levels. The LUNSERS includes 'red-herring' (RH) items designed to identify over-reporting. These items describe phenomena not associated with antipsychotic side effects, e.g. 'A runny nose'. The RH subscale is scored from 0 to 40 , with scores greater than 10 thought to represent high over-reporting (17).

Day et al. (16) examined side effect levels in 50 adult patients with schizophrenia. These patients were taking higher doses of antipsychotic and experiencing moderate levels of side effects on the LUNSERS (scoring 45.1/160 on test and 36.8/160 on retest). These researchers showed the validity of the LUNSERS against the Udvalg for Kliniske Undersogelser (UKU) (18) side effect rating scale, which requires a one-to-one clinical assessment and also demonstrated showed strong concurrent validity between the two measures $(r=0.83, p<0.01)$.
Day et al. (16) further showed the LUNSERS to have good internal reliability (or internal consistency): all side effect items are measuring the same underlying 'side effect construct'. As a general rule, Cronbach's $\alpha$ scores of 0.7 or above show good internal reliability. On test and retest, Day et al. (16) reported strong internal consistency, with Cronbach's $\alpha$ 's of 0.889 on test and 0.886 on retest. The researchers also showed that the LUNSERS has strong stability (or test-retest reliability) over repeated administrations: participants answered LUNSERS items consistently on two testings over a period where the side-effect profile was unlikely to have changed significantly. The test-retest total scores correlated at Pearson's $r=0.81$ and the mean correlation between LUNSERS items was Pearson's $r=0.58$. The mean total $\mathrm{RH}$ score was generally low, with participants averaging 5.1 of $40(\mathrm{SD}=5.3)$ for both test and retest.

Researchers have shown that adult patients suffering from psychotic illnesses are often able to provide valid and reliable self-report of their mental state through survey $(19,20)$. An important question is whether a younger antipsychotic-taking population can provide credible self-reports of side effects. Younger people taking antipsychotics face different challenges in providing self-report than adults. For example, many young people take risperidone for problems associated with disorders such as autism, Asperger's Disorder and subaverage intellectual function $(3,4)$. Therefore, this population may have difficulties with communication and language because of their illnesses as well as their younger age. Furthermore, antipsychotic side-effect profiles may be different in this population and lower doses of antipsychotic medications may sometimes make side effects less apparent.

\section{Box 1. Can young people provide credible self-report of antipsychotic side effects using the LUNSERS? An exploratory study.}

\section{Aims of the study}

It is important to establish whether young people can independently self-report side effects using an established self-report measure like the LUNSERS. With the exception of one article by some of the present authors (21), the LUNSERS has been studied exclusively in adult samples. One means to examine credibility of self-report is to examine the test-retest reliability of the measure within a young sample as compared to an adult sample. Reliable self-report should be associated with coherent and consistent answering across testing. The 
LUNSERS is further suited to this analysis through the use of the RH scale: credible self-report should be associated with low-RH endorsement. Although this analysis does not allow the researchers to ascertain whether side effects were validly identified, a coherent and consistent response pattern provides indirect evidence of credible self-report. This analysis also allows the researchers to examine the use of the LUNSERS as a self-report measure in this younger population.

\section{Method}

\section{Participant inclusion criteria}

Participants were required to be 18 years and under, and taking low-dose risperidone for any condition. To minimise potential fluctuations in side effect levels in those who had recently commenced the drug, participants were required to have taken risperidone for a minimum of 4 months. In order to avoid fluctuations in side effect levels because of the changes in dosage, participants were required to report a stable risperidone dosage during the period of the study. Parents/guardians were allowed to assist the young person through explaining LUNSERS items and helping with survey completion. However, the young person was required to have sufficient language and communication skills to provide the final answers.

\section{Sample description}

Participants were recruited through private child and adolescent psychiatrists and private paediatricians working in Sydney, Australia. The sample comprised 38 participants who were taking risperidone for any condition (26 males and 12 females). The mean age of participants was 12.73 years $(\mathrm{SD}=3.16)$ and ages ranged between 5.8 and 18.3 years. The mean risperidone dose per day was $1.09 \mathrm{mg}(\mathrm{SD}=0.45)$ and ranged between 0.25 and $2.00 \mathrm{mg}$. The mean period of exposure to risperidone was 42.6 months $(\mathrm{SD}=36.2)$ and ranged between 5 and 140 months.

Participants were taking low doses of risperidone for a variety of non-psychotic disorders and this is reflected in the lower doses of risperidone being taken. Although the focus of the study was on side effects rather than diagnosis, the young people generally suffered from Pervasive Developmental Disorders, including autism and Asperger's Disorder, and/or Oppositional/Conduct Disorder with associated aggression (information provided by participating child and adolescent psychiatrists and paediatricians).
All but four of the participants took risperidone in conjunction with other psychotropic medications. For $71 \%$ of the participants, risperidone was prescribed in conjunction with a psychostimulant. The most commonly prescribed psychostimulant was Concerta (long-acting methylphenidate), with 24 participants indicating that they were regularly taking the drug (mean daily dosage $=$ $46.2 \mathrm{mg}, \mathrm{SD}=9.8 \mathrm{mg}$ ). Eight participants were taking antidepressants (six selective serotonin reuptake inhibitor, one serotonin noradrenalin reuptake inhibitor and one tricyclic antidepressant) and one participant was taking an anticonvulsant.

A majority of parents/guardians in the sample indicated that they assisted their child in the completion of the survey.

Procedure

The consent of both parents/guardians and participants was gained before the commencement of the study. The LUNSERS instructions for the study and a reply paid envelope were posted to parents/guardians and participants for completion and return. Approximately 10 weeks after the completion of the first survey, a second LUNSERS survey and a reply paid envelope were posted to the families for completion and return.

Analysis

Missing data were rare except for items in the Hormonal subscale where $74.1 \%$ of the items were missing. This low response rate was because of many of the Hormonal items relating to sexual function that was irrelevant to young participants and marked as optional for ethical reasons. As a result, the researchers decided to exclude the Hormonal subscale from analysis. With the exclusion of the Hormonal subscale, both males and females could report a total score between 0 and 136 on the 'modified' LUNSERS.

A reliability analysis of LUNSERS on test and retest was conducted in a similar manner to that done by Day et al.(16) Descriptive statistics were provided for the side effect totals on the two testings. Cronbach's $\alpha$, as a measure of internal consistency, was calculated for the two testing occasions. To investigate test-retest reliability, Pearson's correlations were performed between corresponding totals, subtotals and scale items on the two testing occasions.

Descriptive statistics were calculated for the $\mathrm{RH}$ subscale on test and retest. In a departure from Day et al.'s (16) analysis, rates of reporting side effects 
were compared to rates of reporting RHs. Because the scales were unequal in size, the proportions of total possible scores were used. The comparison was performed using a paired samples $t$-test. Finally, correlations between RH subscale and total score were calculated on both test and retest.

\section{Results}

LUNSERS totals on test and retest

The mean side-effect total for the first testing was 22.8 of $136(\mathrm{SD}=18.7)$ and ranged from 0 to 61 . The mean side-effect total for the second testing was 22.5 of $136(\mathrm{SD}=16.9)$ and ranged from 0 to 69 .

Internal reliability

Cronbach's $\alpha$ coefficient was high for both testings ( 0.93 for the first testing and 0.91 for the second testing).

Test-retest reliability of LUNSERS totals and side-effect items

Test-retest reliability for the LUNSERS sideeffect total was high $(r=0.82, p<0.001)$. Testretest reliability for the LUNSERS subscales was the lowest for the extrapyramidal subscale $(r=$ $0.60, p<0.001)$ and highest for the miscellaneous subscale $(r=0.97, p<0.001)$.

The mean test-retest reliability for the individual LUNSERS items was $r=0.529$ (range 0.024-0.829) (note that the 'difficulty passing water' item was not used as there was 0 variance as no one selected it). Significant correlations were obtained for all items on the LUNSERS at $p<$ 0.05 with the exception of five items that recorded the correlations of $r<0.3$. These items were 'lack of emotions' $(r=0.16)$, 'sleeping too much' ( $r=$ $0.21)$, 'blurred vision' $(r=0.13)$, 'new/unusual skin marks' $(r=0.08)$ and 'passing a lot of water' $(r=0.02)$.

Red-Herring analysis

The mean total RH score over test and retest was 3.45 of 40 (SD = 4.07). On test and retest, participants reported high-RH (over 10) on a total of 5 of the 76 occasions (6.6\% of the occasions).

For the first testing, a paired sample's $t$-test revealed a significantly greater proportion of side effect items were endorsed than the proportion of RH items endorsed $[M$ side effects $=0.17(\mathrm{SD}=$ $0.14) ; \quad M \mathrm{RH}=0.09(\mathrm{SD}=0.09) ; t(37)=5.52$, $p<0.001]$. This significant result was replicated in the second testing, where a significantly greater proportion of side effect items was endorsed than the proportion of $\mathrm{RH}$ items endorsed $[M$ side effects $=0.17(\mathrm{SD}=0.13) ; M \mathrm{RH}=0.09(\mathrm{SD}=$ $0.11) ; t(37)=5.29, p<0.001]$.

The RH subscale correlated strongly with the LUNSERS total for the first testing $(r=0.75$, $p<0.001)$ and the second testing $(r=0.71, p<$ $0.001)$.

Study limitations

A weakness of this methodology is that it does not allow the researchers to ascertain whether the participants correctly or validly identified side effects; it only permits researchers to examine whether self-report is reliable and seemingly sensible to infer credibility. For example, we cannot be sure whether the young subjects were identifying side effects of their medication or symptoms of their mental illness. Future researchers would need to validate the young person's answers against an independent measure. Furthermore, while the researchers stipulated that the young person provide the answers for the survey, they could not control the extent of parent/guardian involvement in those who did receive assistance. Participants were often taking psychotropic medications other than risperidone, which may have side effects of their own that were recorded on the LUNSERS. This was considered less problematic for the current reliability analysis. Future studies will need to validate self-report against a criterion and control variables such as age and level of adult assistance received. Future studies may wish to examine the type of mental illness from which the young person suffers. This was not done in this study, yet may be important in understanding whether these results apply to all psychiatric disorders, or a subset.

An exploratory study described in Box 1 reports generally lower levels of side effects than did Day et al's (16) sample, consistent with the low doses of risperidone prescribed in this sample. The internal reliability of the LUNSERS as applied to a young sample was high, suggesting that participants were reporting side effects in a systematic way and this accurately reflects the construct being measured. With the exception of five items, test-retest reliability of the LUNSERS in this sample was also high, demonstrating that participants were answering items consistently when retested 3 months later. The young sample generally endorsed a low number of $\mathrm{RH}$ items and the proportion of $\mathrm{RH}$ items endorsed was significantly lower than the proportion for real side-effect items. This provides evidence that 
the sample was able to discriminate genuine side effects from non-related phenomena. However, the strong reliability statistics were tempered by a strong positive correlation between the RH subtotal and the side effect total, suggesting that some participants with high side effect totals may have been overreporting.

The high internal reliability, test-retest reliability and low-RH endorsement provide evidence that the young participants were responding to the LUNSERS in a coherent and sensible manner, which was consistent over separate periods of testing. Participants answered the survey systematically rather than randomly endorsing items or endorsing all items. These results provide indirect evidence that young participants were generally able to give credible and sensible self-report of side effects using the LUNSERS when provided with a level of assistance.

Reliability statistics from this younger sample are comparable to those reported by Day et al. (16) in a group of adults suffering from schizophrenia. Yet, a direct comparison cannot be made between the groups, because even though the younger participants may have experienced greater difficulties with communication associated with their age and/or illness, they were unlikely to have had the same degree of cognitive deficit resulting from psychotic symptoms and/or higher antipsychotic dosage as the adult group. Therefore, the researchers can only conclude that both groups produced comparable data demonstrating high reliability in spite of their respective difficulties.

\section{Strengths and weaknesses of using the LUNSERS for young people}

The coherent and consistent self-report of the young participants also attests to the value of the LUNSERS as a side-effect measure in this population. The measure showed reliability and robustness in the younger population. The RH subscale appeared useful in identifying over-reporting. Furthermore, the researchers anecdotally observed a more proactive collaboration between young people and parents in the monitoring of side effects as a result of having to complete the LUNSERS regularly. Most families originally were unaware of the variety of side-effect risks. An unintended consequence of the study was that regular completion of the LUNSERS fostered greater awareness of and vigilance towards potential side effects. For these reasons, there may be value in the use of a self-report measure like the LUNSERS for the monitoring and measurement of side effects in young people taking antipsychotic medication.

However, survey measures are often associated with a loss of information and qualitative data that can be gained through a comprehensive one to one examination like the UKU should be considered. The researchers in this study noted some aspects of the LUNSERS that were potentially problematic for use in the general population, as well as difficulties specific to its use in young people. For example, 16 of the LUNSERS' 40 side effect items were also the symptoms of mental illness, e.g. 'depression'. This overlap could result in people who are unwell recording higher LUNSERS scores. By contrast, many of the side effect items also describe more general phenomena experienced by people not taking antipsychotic medications. For example, the low test-retest reliability for the items 'new and unusual skin marks' and 'sleeping too much' may reflect the characteristics of a teenage sample that periodically sleeps excessively and suffers from acne.

Total scores on the LUNSERS should be interpreted cautiously as these may not be representative of subjective discomfort or distress. For example, a single side effect item marked 'very much' may warrant discontinuation of medication despite producing a low-total score of 4/160. Therefore, the LUNSERS may be useful for gaining an overall prevalence profile of possible side effects, as suggested by Morrison et al. (22), rather than being considered a quantitative measure of side effect induced discomfort.

Some aspects of the LUNSERS may need refinement if it were to be used in a young population. Scale items may need simple yet descriptive language and/or pictures to help the young people identify side effects. The low test-retest reliability on some items is possibly because of language with which young people may be unfamiliar, e.g. 'passing water'. Furthermore, researchers and clinicians would need to consider how best to sensitively ask questions related to sexual function to post-pubescent patients and whether it is appropriate to ask these questions in a survey format. It is yet to be established whether young patients can distinguish between side effects and symptoms, suggesting that self-report measures like the LUNSERS may be useful in gaining a general and inexact side effect profile, but cannot substitute for a thorough side-effect assessment from a clinician.

Clinicians and researchers rely upon accurate selfreport of antipsychotic side effects from their patients and study participants. The young sample from this study provided sensible self-report with no more than a paper and pencil measure and a basic level of assistance. These results suggest that young people taking low-dose antipsychotics with basic language skills are able to report and rate their side effects in spite of the challenges posed by their age and illness. There is thus preliminary evidence that in many cases the quality of self-report ratings should 
not be underestimated. Furthermore, the LUNSERS can be a valuable general measure for monitoring and managing side effects in young people. However, results of self-report measures like the LUNSERS are only meaningful within the context of a comprehensive side effect assessment by a clinician.

\section{Acknowledgements}

The study was funded by a Northern Sydney Area Collaborative Research Grant. We thank the families who volunteered for this research without them it would not have been possible. We are appreciative of their patience and generosity. We also thank the NHMRC Program Grant 510135.

\section{Disclosure}

Garry Walter has received educational grants from Eli Lilly, Janssen-Cilag and Pfizer, and travel assistance and an honorarium for a talk from Eli Lilly. Glenn Hunt has received educational grants from Eli Lilly and Pfizer. Michelle Cleary has received educational grants from Eli Lilly, Janssen-Cilag and Pfizer. Paul Duffy has received educational grants from Eli Lilly, Janssen-Cilag and Novartis in the form of travel sponsorship to conferences. Geoff Crawford has received travel grants from Janssen-Cilag and Eli Lilly, and honoraria for talks by Janssen-Cilag and Eli Lilly. Gin Malhi has served on pharmaceutical advisory boards, received funding for research and been in receipt of honoraria for talks at sponsored meetings involving AstraZeneca, Eli Lilly, JanssenCilag, Organon, Pfizer and Wyeth. Patrick Concannon has been on the advisory board of Novartis and Janssen-Cilag. The other authors have nothing to disclose.

\section{References}

1. FindLING RL. Atypical antipsychotics in the treatment of children and adolescents. J Clin Psychiatry 2004;65(Suppl. 6):3-29.

2. Olfson M, Blanco C, Liu L, Moreno C, Laje G. National trends in the outpatient treatment of children and adolescents with antipsychotic drugs. Arch Gen Psychiatry 2006;63:679-685.

3. Cheng-Shannon J, McGough MD, Pataki C, MCCRACKEN JT. Second-generation antipsychotic medications in children and adolescents. J Child Adolesc Psychopharmacol 2004;14:372-394.

4. Walter G, Delaroche A, Soh N et al. Side effects of second generation antipsychotics: the experience, views and monitoring practices of Australian child psychiatrists. Aust Psychiatry 2008;16:253-262.

5. Silvaprasad L, Hassan T, Handy S. Survey of atypical antipsychotic medication used by children and adolescent psychiatrists. Child Adolesc Ment Health 2006;11:164-167.

6. Doey T, Handelman K, Seabrook JA, Steele M. Survey of atypical antipsychotic prescribing by Canadian child psychiatrists and developmental pediatricians for patient age under 18 years. Can J Psychiatry 2007;52:363-368.

7. Efron D, Hiscock H, Sewell JR et al. Prescribing of psychotropic medications for children by Australian pediatricians and child psychiatrists. Pediatrics 2003;111:372-375.

8. Croonenberghs J, Fegert J, Findling RL, De Smedt G, VAN Dongen S. Risperidone in children with disruptive behavior disorders and subaverage intelligence: a 1-year, open-label study of 504 patients. J Am Acad Child Adolesc Psychiatry 2005;44:64-72.

9. Reyes M, Croonenberghs J, Augustyns I, Eerdekens M. Long-term use of risperidone in children with disruptive behavior disorders and subaverage intelligence: efficacy, safety and tolerability. J Child Adolesc Psychopharmacol 2006;16:260-272.

10. Reyes M, Olah R, Csaba K, Augustyns I, Eerdekens M. Long-term safety and efficacy of risperidone in children with disruptive behaviour disorders: results of a 2-year extension study. Eur Child Adolesc Psychiatry 2005;15:97-104.

11. Correll CU. Metabolic side effects of second-generation antipsychotics in children and adolescents: a different story. J Clin Psychiatry 2005;66:1331-1332.

12. Correll CU. Endocrine and metabolic adverse effects of psychotropic medications in children and adolescents. J Am Acad Child Adolesc Psychiatry 2006;45:771-791.

13. Correll CU. Antipsychotic use in children and adolescents: minimizing adverse effects to maximise outcomes. J Am Acad Child Adolesc Psychiatry 2008;47:9-20.

14. Awad AG, Voruganti LN. Intervention research in psychosis: issues related to the assessment of quality of life. Schizophr Bull 2000;26:557-564.

15. Aman MG, Arnold E, McDougle CJ et al. Acute and long-term safety and tolerability of risperidone in children with autism. J Child Adolesc Psychopharmacol 2005;15:869-884.

16. Day JC, Wood G, Dewey M, Bentall RP. A Self-Rating Scale for Measuring Neuroleptic Side-Effects - validation in a group of schizophrenic patients. $\mathrm{Br} \mathrm{J}$ Psychiatry 1995;166:650-653.

17. LAMBERT TJR. Measurement of antipsychotic-induced sideeffects: support for the validity of a self-report (LUNSERS) versus structured interview (UKU) approach to measurement. Hum Psychopharmacol 2003;18:405-411.

18. Linjaerde O, Ahlfors UG, Bech P, Dencker SJ, Elgen $\mathrm{K}$. The UKU side effect rating scale. A new comprehensive rating scale for psychotropic drugs and a cross-sectional study of side effects in neuroleptic treated patients. Acta Psychiatr Scand 1987;76:1-100.

19. Voruganti L, Heslegrave R, Awad AG, Seeman MV. Quality of life measurement in schizophrenia: reconciling the quest for subjectivity with the question of reliability. Psychol Med 1998;28:165-172.

20. Amador XF, Strauss DH, Yale SA, Flaum MM, Endicott J, Gorman JM. Assessment of insight in psychosis. Am J Psychiatry 1993;150:873-879.

21. Byrne S, Walter G, Hunt G et al. Duration of treatment and self-reported neuroleptic side-effects of low dose risperidone in children and adolescents. Aust Psychiatry 2010; 18:42-45.

22. Morrison P, Gaskill D, Meehan T, Lunney $P$, Lawrence G, Collings P. The use of the Liverpool University Side Effect Rating Scale (LUNSERS) in clinical practice. Aust N Z J Ment Health Nurs 2000;9:166-176. 